\title{
Table of cases (in chronological order)
}

\section{ECJ/CJEU}

Joined Cases C-28/62, C-29/62 and C-30/62 Da Costa en Schaake NV, Jacob Meijer $N V$, Hoechst-Holland NV v Netherlands Inland Revenue Administration [1963] 00061 101

Case C-78/70 Deutsche Grammophon Gesellschaft mbH v Metro-SB-Großmärkte GmbH \& Co KG [1971] 00487

Case C-62/79 SA Coditel Brabant and Others v S.A. Ciné Vog Films and Others ('Coditel I') [1980] 00881

Case C-57/80 Musik-Vertrieb membran GmbH and K-tel International v GEMAGesellschaft für musikalische Aufführungs und mechanische Vervielfältigungsrechte [1981] ECR 147 136,142

Case C-10/89 SA CNL-SUCAL NV v Hag GF AG [1990] I-03711 141

Joined Cases C-92/92 and C-326/92 Phil Collins v Imtrat Handelsgesellschaft mbH and Patricia Im-und Export Verwaltungsgesellschaft mbH and Leif Emanuel Kraul v EMI Electrola GmbH [1993] I-05145

Case C-9/93 IHT Internationale Heitechnik GmbH and Uwe Danziger v Ideal Standard GmbH and Wabco Standard GmbH [1994] I-02789

Joined Cases C-241/91 P and C-242/91 P Radio Telefis Eireann (RTE) and Independent Television Publications Ltd (ITP) $v$ Commission of the European Communities [1995] I-00743

Case C-398/95 Syndesmos ton en Elladi Touristikon kai Taxidiotikon Grafeion (SETTG) v Ypourgos Ergasias [1997] I-03091

Case C-61/97Foreningen af danske Videogramdistributørerv Laserdisken [1998]

Case C-60/98 Butterfly Music Srl v Carosello Edizioni Musicali e Discografiche (CEMED) [1999] I-03939

Case C-6/98 Arbeitsgemeinschaft Deutscher Rundfunkanstalten (ARD) v PRO

Sieben Media AG [1999] I-07599 _..................................................... 136

Case C-23/99 Commission v France [2000] I-07653 ....................................... 141

Case C-280/00 Altmark Trans GmbH and Regierungspräsidium Magdeburg v

Nahverkehrsgesellschaft Altmark GmbH [2003] I-07747 ........................ 100

Case C-115/02 Administration des douanes et droits indirects $v$ Rioglass SA and

Transremar SL [2003] I-12705

Case C-46/02 Fixtures Marketing Ltdv Oy Veikkaus Ab [2004] I-10365 .... 158, 166

Case C-203/02 The British Horseracing Board Ltd and Others $v$ William Hill

Organization Ltd [2004] I-10415 $157,158,166$

Case C-338/02 Fixtures Marketing Ltd v Svenska Spel AB [2004] I-10497 ....... 158, 
Case C-444/02 Fixtures Marketing Ltd v Organismos prognostikon agonon podosfairou AE (OPAP) [2004] ECR I-10549 $158,164,166$

Case C-192/04 Lagardère Active Broadcast v Société pour la perception de la rémunération équitable (SPRE) and Others [2005] I-07199 142,232

Joined Cases C-94/04 and C-202/04 Federico Cipolla v Rosaria Fazari, née Portolese, and Stefano Macrino and Claudia Capodarte v Roberto Meloni [2006] I-11421 100,136

Case C-306/05 Sociedad General de Autores y Editores de España (SGAE) v Rafael Hoteles SA [2006] I-11519 104

Case C-250/06 United Pan-Europe Communications Belgium SA and Others v État belge [2007] I-11135 136

Case C-304/07 Directmedia Publishing GmbH v Albert-Ludwigs-Universität Freiburg [2008] I-07565

Case C-52/07 Kanal 5 Ltd and TV 4 AB v Föreningen Svenska Tonsättares Internationella Musikbyrå (STIM) upa [2008] I-09275

Case C-222/07Unión de Televisiones Comerciales Asociadas (UTECA) v Administración General del Estado [2009] I-01407

Case C-545/07 Apis-Hristovich EOOD v Lakorda AD [2009] I-01627 158

Case C-478/07 Budějovický Budvar, národní podnik v Rudolf Ammersin GmbH [2009] I-07721

Case C-5/08 Infopaq International A/S v Danske Dagblades Forening [2009] I-06569 ........4, 90, 95, 99, 105, 107, 120, 151, 159, 166, 170, 180, 185, $186,190,197,199$

Case C-42/07 Liga Portuguesa de Futebol Profissional and Bwin International Ltd v Departamento de Jogos da Santa Casa da Misericórdia de Lisboa [2009] ECR I-07633

Case C-393/09 Bezpečnostní softwarová asociace - Svaz softwarové ochranyv Ministerstvo kultury [2010] I-13971 4, 100, 122, 166, 179, 185, 206

Case C-168/09 Flos SpA v Semeraro Casa e Famiglia SpA [2011] I-00181 ....... 109

Joined Cases C-403/08 and C-429/08 Football Association Premier League Ltd, NetMed Hellas SA, Multichoice Hellas SA v QC Leisure, David Richardson, AV Station plc, Malcolm Chamberlain, Michael Madden, SR Leisure Ltd, Philip George Charles Houghton, Derek Owen and Karen Murphy v Media Protection Services Ltd .... 4, 129, 136, 137, 140, 141, 142, 146, 152, 166, 206

Case C-145/10 Eva-Maria Painer v Standard VerlagsGmbH, Axel Springer AG, Süddeutsche Zeitung GmbH, SPIEGEL-Verlag RudolfAUGSTEIN GmbH \& Co KG and Verlag M. DuMont Schauberg Expedition der Kölnischen Zeitung $\mathrm{GmbH} \& \mathrm{Co} \mathrm{KG}$ 4, 148, 151, 152, 166, 190, 197, 200, 206

Case C-604/10 Football Dataco and Others v Yahoo! UK Ltd and Others ..... 5, 125 , $155,167,200$

Case C-135/10 Società Consortile Fonografici (SCF) v Marco Del Corso ........ 147 Case C-162/10 Phonographic Performance (Ireland) Limited v Ireland, Attorney

General 147

Case C-510/10 DR and TV2 Danmark A/S v NCB - Nordisk Copyright Bureau ... 18 Case C-406/10 SAS Institute Inc $v$ World Programming Ltd Case C-5/11 Criminal proceedings against Titus Donner $5,174,183,185$ 2 
Case C-521/11 Amazon.com and Others v Austro-Mechana Gesellschaft zur Wahrnehmung mechanisch-musikalischer Urheberrechte Gesellschaft $\mathrm{mbH}$

Case C-466/12 Nils Svensson, Sten Sjögren, Madelaine Sahlman, Pia Gaddv Retreiver Sverige $A B$

\section{Belgium}

Artetessuto $n v v B \&$ T Textilia $n v$ and Indecor-Europe $n v$, Hof van Cassatie van België, arrest No C.11.0108.N, 26 January 2012

\section{Canada}

Slumber-Magic Adjustable Bed Co v Sleep-King Adjustable Bed Co and Others (1984) 3 CPR (3d) 81 (BCSC)

CCH Canadian Ltd v Law Soc'y of Upper Can [2004] 1 SCR 339 60,171

\section{France}

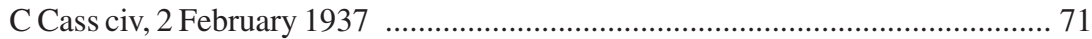

Tribunal de commerce Seine, 26 June 1951 ......................................................... 71

Court d'appel Paris, 4e ch, 30 April 1963 .............................................................. 71

C Cass civ 1re, 1 July 1970: D 1970, 734 ......................................................... 139

C Cass civ 1re, 13 November 1973 ................................................................. 139

Cour d'appel Lyon, 5 July 1979 ...................................................................... 71

Babolat Maillot Witt v Pachot, C Cass, Assemblée plénière, 7 March 1986, No 82

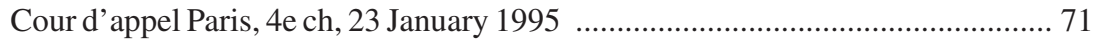

Tribunal de Grande Instance de Nanterre, 28 April 1998 ...................................... 71

Cour d'appel Paris, 4e ch, 16 September 2005 ...................................................... 71

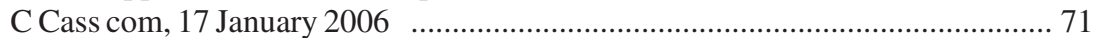

Cour d'appel Paris, Groupe Liaisons v Kavesta, PIBD 839111720, 2 June

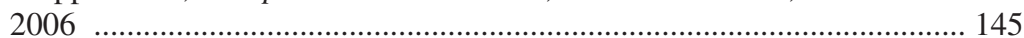

C Cass, civ 1re, 20 October 2011 (pourvoi No 10-21.251) ................................. 198

Philippe G and Alexandra Jv Paul M, Tribunal de grande instance de Paris, 3ème ch, 4ème sec, 20 December 2012

\section{Germany}

Inkassoprogramm, BGH GRUR 1985, 1041/1047 ................................. 64, 139

Anwaltschriftsatz, BGH GRUR 1986, 739/740 …...................................... 139

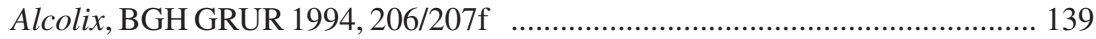

Stadtplanwerk, BGH GRUR 1998, 916/917 .................................................. 139

Pharma Intranet Information AG v IMS Health GmbH \& Co OHG [2005]

ECC 12

\section{Italy}

British American Tobacco Italia SpA v Oneoffcigars Ltd and Vanderwood Italia Srl, Tribunale di Roma, No 25016, 20 December 2010 
Burberry Ltd v Profumerie Frediani Snc and A L Team Srl, Tribunale di Firenze,

No 2348, 27 June 2011

RTI Reti Televisive Italiane and Another $v$ Rai and Another, Tribunale di Roma,

21 October 2011

Flos SpA v Semeraro Casa \& Famiglia SpA, Tribunale di Milano, 12 September 2012

RTI-Mediaset v dinozap.tv and Others, Procura di Milano, 11 January 2013 ..... 141

\section{New Zealand}

Wham-O Manufacturing Co v Lincoln Industries Ltd [1985] RPC 127 ............. 203

Breville Europe Plc v Thorn EMI Domestic Appliances Ltd [1995] FSR 77 ....... 203

Metix (UK) Ltd v G H Maughan (Plastics) Ltd [1997] FSR 718 .......................... 203

Wildash v Klein (2004) 61 IPR 324

\section{Slovak Republic}

Ecopress as v Storin, spol sro, 11Co 51/2010-447 IČS: 1308209396, 27 September 2011

\section{United Kingdom}

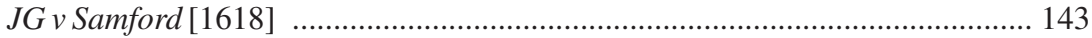

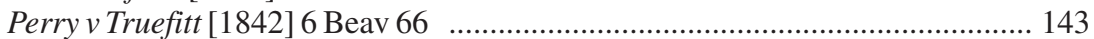

Graves' Case [1869] LR 4 QB 715 .......................................................... 94, 196

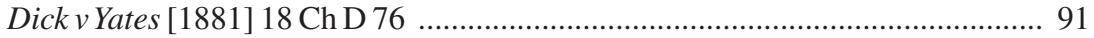

Reddaway v Banham [1896] AC 199; 13 RPC 218 ......................................... 143

Fournet v Pearson Ltd (1897) 14 TLR 82 ...................................................... 77

London Tramways v London County Council [1898] AC 375 ........................... 101

Walter v Lane [1900] AC 539 ................................................................... 89, 91

University of London Press, Ltd v University Tutorial Press, Ltd [1916]

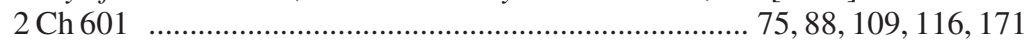

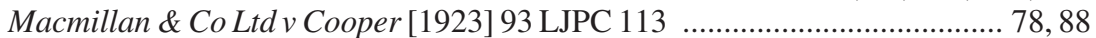

Francis Day and Hunter v Twentieth Century Fox Corp and Others [1939]

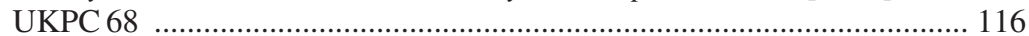

Football League Ltd v Littlewoods Pools [1959] 1 Ch 637 ............................... 163

Ladbroke (Football) Ltd v William Hill (Football) Ltd [1964] 1 WLR 273 .... 67, 78, 93, 145

George Hensher Ltd v Restawile Upholstery (Lancs) Ltd [1976] AC 64 ............ 203

Bauman v Fussell [1978] RPC 485 ................................................................ 191

Erven Warnink BV v Towend (J) \& Sons [1979] AC 731 ................................... 144

Exxon Corp v Exxon Insurance Consultants International Ltd [1981] 1 WLR 624;

[1981] 2 All ER 495; 3 All ER 241

89,117

Interlego v Tyco Industries [1989] AC 217 .................................. 90, 94, 193, 197

Express Newspaper Plc v News (UK) Ltd [1990] FSR 359 (Ch D) ...................... 88

Reckitt \& Colman v Borden [1990] RPC 340 HL .............................................. 143

Total Informatics Processing Systems Ltd v Daman Ltd [1992] FSR 171 ............ 177

Creation Records Ltd and Others v News Group Newspapers Ltd [1997] EWHC Ch 370 198

Designers Guild, Ltdv Russell Williams (Textiles), Ltd, [2000] 1 WLR 2416 ...... 92 
The Newspaper Licensing Agency Ltd v Marks \& Spencer [2001] UKHL 38 .....92,

Antiquesportfolio.com v Rodney Fitch \& Co Ltd, [2001] FSR 345; [2001] ECDR 51

IPC Media v Highbury-Leisure Publishing [2004] FSR 20

Sawkins v Hyperion Records Ltd [2005] EWCA Civ 565

Navitaire Inc v easyJet Airline Company and Bulletproof Technologies Inc [2004]

EWHC 1725 (Ch); [2006] RPC 4

176,180

Coffey v Warner/Chappell Music [2005] EWHC 449 (Ch); [2005] ECDR 21; [2006]

EMLR 2; [2005] FSR 34; (2005) 28(6) IPD 28046

$O$ (Peter) v F KG [2006] ECDR 9

Nova Productions Ltd v Mazooma Games Ltd and Others [2006] EWHC 24 (Ch);

[2007] EWCA Civ 219

177,178

Karen Murphyv Media Protection Services Ltd [2007] EWHC 3091 (Admin);

[2007] EWHC 529 (Admin)

SAS Institute Inc $v$ World Programming Ltd, [2010] EWHC 1829 (Ch) ............ 174

Football Dataco Ltd and Others $v$ Brittens Pools Ltd and Others, [2010] EWHC 841

(Ch); [2010] EWCA Civ 1380 125,156

Paul Gregory Allen (acting as trustee of Adrian Jacobs (deceased)) v Bloomsbury

Publishing Plc and Joanne Kathleen Murray (professionally known as JK

Rowling) [2010] EWHC 2560 (Ch)

Football Association Premier League Ltd, NetMed Hellas SA, Multichoice Hellas SA v QC Leisure, David Richardson, AV Station plc, Malcolm Chamberlain, Michael Madden, SR Leisure Ltd, Philip George Charles Houghton, Derek Owen [2008] EWHC 1411 (Ch); [2012] EWHC 108 (Ch)

Lucasfilm Ltd and Others $v$ Andrew Ainsworth and Another [2008] EWHC 1878

(Ch); [2009] EWCA Civ 1328; [2011] UKSC 39 6, 125, 203, 204, 205

The Newspaper Licensing Agency Ltd and Others $v$ Meltwater Holding BV and Others [2010] EWHC 3099 (Ch); [2011] EWCA Civ 890 .... 6, 111, 113, 114,

Football Dataco Ltd, Football Association Premier League Ltd, Football League Limited, Scottish Premier League Ltd, Scottish Football League and PA Sport UK Ltd v Yahoo! UK Limited, Stan James (Abingdon) Limited, Stan James PLC and Enetpulse APS, [2010] EWCA Civ 1380 156,157

Future Publishing Limited $v$ The Edge Interactive Media Inc and Others [2011] EWHC 1489 (Ch)

Forensic Telecommunications Services Ltd $v$ The Chief Constable of West Yorkshire

Police Stephen Hirst [2011] EWHC 2892 (Ch) 124

The Queen on the application of British Telecommunication Plc and TalkTalk

Telecom Group Plc v Secretary of State for Culture, Olympics, Media and Sports and Others [2012] EWCA Civ 232

Football Association Premier League Ltd and Others $v$ QC Leisure and Others

[2012] EWHC 108 (Civ)

Karen Murphy v Media Protection Services Ltd [2012] EWHC 466 (Admin) ... 148

Football Dataco and Others $v$ Sportradar GMBH and Another and Football Dataco and Others $v$ Stan James Abingdon Ltd and Others [2012] EWHC 1185

(Ch) 
Temple Island Collections Ltd v New English Teas Ltd and Nicholas John Houghton [2012] EWPCC 1 6, 190, 191, 192, 193, 194

Abraham Moon \& Sons Ltd v Andrew Thornber and Others [2012]

EWPCC 37 205

Football Association Premier League Ltd v QC Leisure and Others [2012] EWCA

Civ 1708

SAS Institute Inc v World Programming Ltd [2013] EWHC 69 (Ch) 186

Public Relations Consultants Association Limited v The Newspaper Licensing Agency Limited and others [2013] UKSC 18

\section{United States}

Clayton v Stone, 5 F Cas 999, 1000, CCSDNY [1829]

Emerson v Davies, 8 F Cas 615, CCD Mass [1845] 84,86

Jolly v Jacques, 13 F Cas 910, CCSDNY [1850] 85

Trademark cases, 100 US 82 [1879] 84,85

Burrow-Giles Lithographic Co v Sarony, 111 US 53 [1884]

Bleistein v Donaldson Lithographic Co, 188 US 239 [1903] $84,87,152$

Alfred Bell \& Co Ltd v Catalda Fine Arts, Inc, 191 F2d 99, $2^{\text {nd }}$ Cir [1951] ........... 87

Sony Corp of Am v Universal City Studios, Inc, 464 US 417 [1984] .................... 214

Feist Publications, Inc, v Rural Telephone Service Co, 499 US 340 [1991] ... 60, 71, 73,79

Key Publications, Inc v Chinatown Today Publishing Enters 945 F 2d 509, 511, $2^{\text {nd }} \operatorname{Cir}[1991]$

The Bridgeman Art Library Ltd v Corel Corp, 25 F Supp 2d 421, 36 F Supp 2d 191 [1998] 94, 196

Reed-Elsevier v Muchnick, 130 S Ct 1237, 1241 [2010] .................................. 212

Peters $v$ West and Others, 11-1708, $7^{\text {th }}$ Cir [2012] 